\title{
Is there any relationship between autism and pineal gland volume?
}

\author{
Feride F. Görgülü̈A,B,D,D,F, Ayşe S. Koç,A,D,E,F \\ Department of Radiology, University of Health Sciences, Adana Health Practice and Research Center, Adana, Turkey
}

\section{Abstract}

Purpose: Abnormalities in melatonin physiology and circadian rhythm are detected in patients with autism. Melatonin is produced predominantly in the pineal gland and the amount of melatonin released is proportional to the pineal gland volume. This study aimed to examine whether the pineal gland volume in children with autism is different from that in healthy children.

Material and methods: Brain magnetic resonance images (MRI) of 120 paediatric patients with autism and 82 control paediatric subjects were examined; pineal parenchymal volume (PPV), pineal cyst rate (PCR), and total pineal gland volume (TPGV) were measured using a multimodality viewer (MMV), but only the TPGVs were measured using a tumour tracking (TT) method. Measurements were taken by 2 separate radiologists.

Results: In patients with autism, the PPV and TPGV according to MMV, and the TPGV according to TT were significantly lower, and the PCR was significantly higher. Moreover, the ratio of PPV to TPGV was significantly lower in the autism patient group. In both groups, the TPGVs were significantly lower in the autism patient group than the controls among all age groups.

Conclusions: Our study was the first to examine TPGVs in detail in paediatric patients with autism using 2 different methods. Low PPV-TPGV and high PCR have been observed in autism. This study also provides comparable reference values for pineal gland size in healthy children or autistic children aged 2-17 years. These results show promising potential for further research to understand the relationship between autism pathogenesis and the pineal gland.

Key words: pineal gland, autism spectrum disorder, children, brain MRI, pineal parenchyma.

\section{Introduction}

Autism spectrum disorder (ASD) is a complex neurodevelopmental disorder that is either congenital or occurs in the first 3 years of life, affecting $1 \%$ of the general population. The disease is characterized by disorders in communication and social interaction, accompanied by restrictive and repetitive behaviour [1].

Abnormalities in melatonin physiology and circadian rhythm are detected in ASD patients. The physiological levels of melatonin and/or melatonin derivatives are generally below average in ASD, and they are correlated with autistic behaviour. In children with autism, these abnormalities in melatonin concentrations have been reported to be due to variations in the melatonin production pathway and genetic pathways. The relationship between daytime behaviours and better night-time management of children with ASD who underwent exogenous treatment with melatonin supplements was previously reported [2,3]. It has been observed that approximately $65 \%$ of patients with ASD have less than half the mean melatonin values. Furthermore, sleep disturbance is seen in $9-50 \%$ of normal children, but it is one of the most common symptoms in paediatric ASD patients, with a prevalence of $50-80 \%$ [4]. Melatonin is an endogenous neuro-hormone that is produced predominantly in the dark, at night in the pineal gland, and the amount of melatonin released is proportional to the volume of the pineal gland [5].

Correspondence address:

Feride Fatma Görgülü, Assistant Professor, MD, Department of Radiology, University of Health Sciences, Adana Health Practice and Research Center, Adana,

Turkey, e-mail:drferide@yahoo.com

Authors' contribution:

A Study design · B Data collection · C Statistical analysis · D Data interpretation · E Manuscript preparation · F Literature search · G Funds collection 
The pineal gland is a photo-neuro-endocrine gland protruding from the posterior wall of the third ventricle in the centre of the brain. Its position enables it to provide direct secretion to the cerebrospinal fluid and to secret hormones into the blood [1]. The pineal parenchyma volume (PPV) (corrected pineal volume for individuals with pineal cysts), which represents the part that releases melatonin, can be considered to be proportional to the number and total volume of pinealocytes. Moreover, pineal cysts, which are very common but whose effects on pineal function are unknown, should also be considered in order to achieve the correct result when analysing the function of this gland $[7,8]$.

This present study aimed to examine whether the total or parenchymal volume of the pineal gland in children with ASD is different from that in healthy children. Our second aim was to examine whether there is a difference between patients with ASD and control individuals in terms of the presence of a pineal gland cyst. Our third target was to investigate whether there is a change in pineal gland volume (PGV) based on increasing age groups in ASD children and normal children. Finally, in light of the fact that knowing the normal pineal gland volume can help radiologists to identify abnormal cases, we also aimed to reveal normal pineal gland volumes in children, which could be used to compare MRI findings.

\section{Material and methods}

\section{Study population}

A total of 120 children with definitively diagnosed ASD and 82 control paediatric subjects, with both groups aged 2-17 years, from data obtained from Adana City Research and Training Hospital (Adana, Turkey), were included in this study. Patients diagnosed with ASD registered in the hospital database, who had undergone brain magnetic resonance imaging (MRI) and were between the age

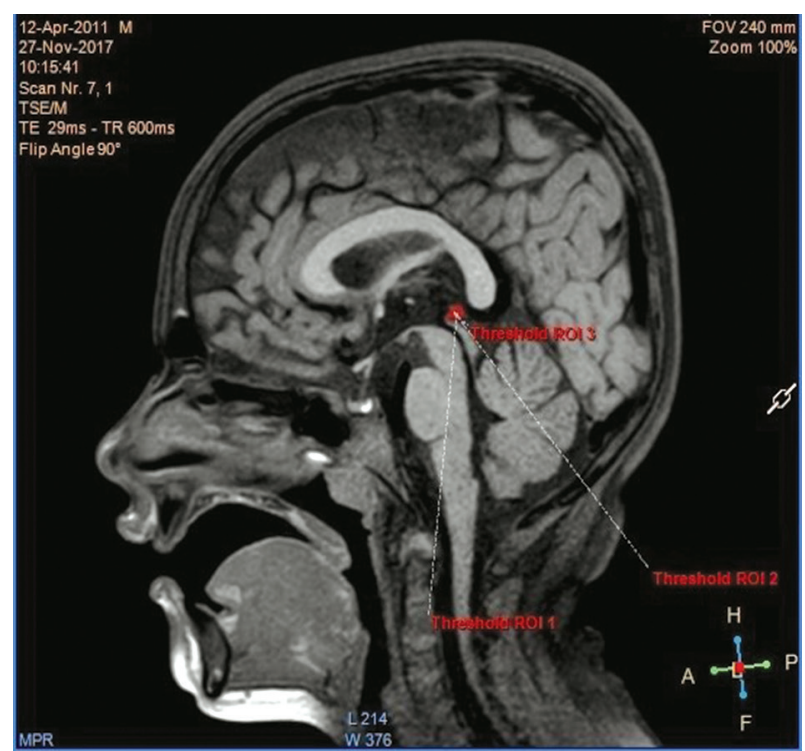

Figure 1. Total pineal gland volume measurement of a 6 -year-old male patient using the multimodality viewer method of 2 and 17 years, were included in the study. The PGVs of the paediatric patients from whom brain MRIs were taken for various reasons and evaluated as normal in similar age groups were selected as the control group. Patients and control subjects with pineal calcification, with history of a brain trauma, operation, and tumour history were excluded from the study. The patients' parents gave their signed informed consent, which also provided them with a comprehensive description of the study. All procedures were approved by the Ethics Committee of Adana City Research and Training Hospital prior to initiation of this study.

\section{Volumetric analysis}

All the MRI studies were performed without contrast administration on the same 3.0-Tesla scanner (Philips Medical Systems, Ingenia, Netherlands). We used the corpus callosum and the upper colliculus, the posterior part of the third ventricle, and the quadrigeminal cistern as the anatomical landmarks to define the pineal gland. We marked and measured the volume of the pineal glands primarily in the sagittal planes and supported the results in axial and coronal planes. Volumetry of the pineal gland and pineal cysts was done using the volume analysis program, and 3-dimensional (3-D) volumetric analysis was performed on a workstation using Philips Intellispace Portal software. There are 2 different volume measurement software in this program, one is a multimodality viewer (MMV) and the other is a tumour tracking (TT) method. The pineal gland parenchyma, cyst, and total gland volumes were measured in T1-weighted sequences using the MMV method (Figure 1). When describing the pineal cyst, we considered it to be a homogeneous area isointense to cerebrospinal fluid in T1-weighted images (Figure 2). Becuase it is technically difficult to distinguish between a pineal cyst and the parenchyma with TT, only total PGVs were measured in T1-weighted sequences using the TT method (Figure 3). The PPV was calculated by subtracting the volume of the

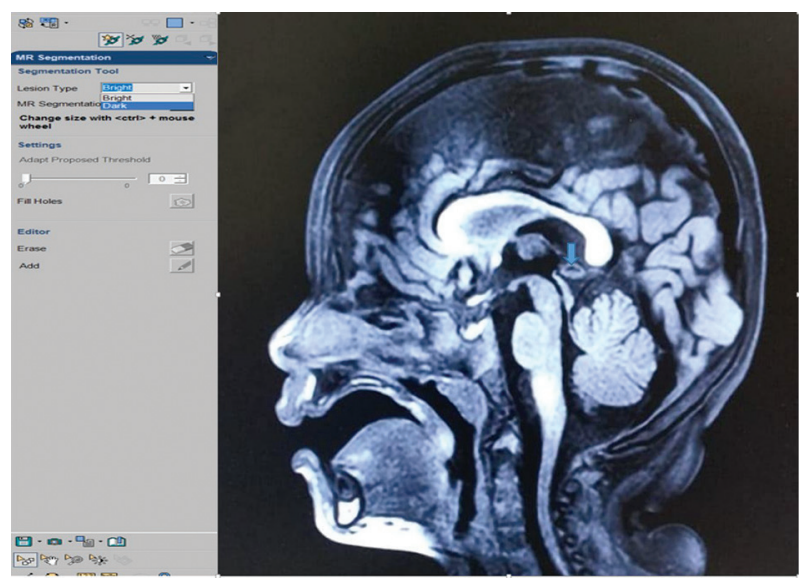

Figure 2. In 3-dimensional T1-weighted sagittal section, pineal parenchyma was measured in manual bright selection by multimodality viewer method in magnetic resonance segmentation, while cyst volume was measured in dark selection. Blue arrow shows pineal cyst in a healthy 3-year-old child 
cyst from the total pineal volume, considering that pineal cysts do not contain pinealocytes. All volumetric data were given as means \pm standard deviation (SD) in cubic $\mathrm{mm}$ from 2 different measurements that had a 1-week time gap between 2 measurements. The final value was determined by taking the average.

The sequences of the study protocol images used in the volumetric measurements consisted of 3-D Brainview T1W, TSE (turbo spin echo) T2W - coronal, TSE T2W - axial, T1WIR (inversion recovery) - coronal, and Flair-SPIR (spectral pre-saturation with inversion recovery)-axial. The file format was DICOM. The slice thicknesses were 1-3 mm. The study protocol of 3-D structural T1-weighted gradient echo sequences consisted of the following parameters: flip angle $=90^{\circ}$; field of view $=240 \times 240 \mathrm{~mm}$; repetition time $=600 \mathrm{~ms}$; echo time $=29 \mathrm{~ms}$; acquired voxel size $=0.99$ $\times 1.0 \times 1.0 \mathrm{~mm} ; 1.0 \mathrm{~mm}$ sagittal slice thickness without interslice gap. Parameters of TSE (turbo spin echo) T2-weighted sequences were detailed as follows: acquisition voxel size = $0.70 \times 0.82 \times 3.0 \mathrm{~mm} ; 3.0 \mathrm{~mm}$ sagittal slice thickness with no inter-slice gap; repetition time $=4.2 \mathrm{~ms}$; echo time $=103 \mathrm{~ms}$; flip angle $=90^{\circ}$; field of view $=240 \times 240 \mathrm{~mm}$.

Measurements were taken by 2 separate radiologists (FFG, ASK), who were unaware of the patients' clinical diagnosis, using 1 of 2 methods; one method used a multimodality viewer (MMV) by FFG and the other used tumour tracking (TT) by ASK. Both of the radiologists who took the measurements had 15 years of experience.

\section{Statistical analysis}

The Shapiro Wilk test was used to determine the normality of the continuous variables. Because the data showed normal distribution, Student's t test was used to determine the average differences between the ASD patients and the control group. Pearson's correlation coefficients were calculated to determine the linear relationship between total pineal gland volume (TPGV)-MMV $\left(\mathrm{mm}^{3}\right)$ and TPGV-TT $\left(\mathrm{mm}^{3}\right)$ in the patient and control groups. To observe the compatibility between the TT and MMV techniques, the in-class correlation coefficient was calculated. Receiver operating characteristic (ROC) analysis was used to determine the cut-off point for TT and MMV and to compare the area

Table 2. Comparison of the distribution of the studied parameters by group

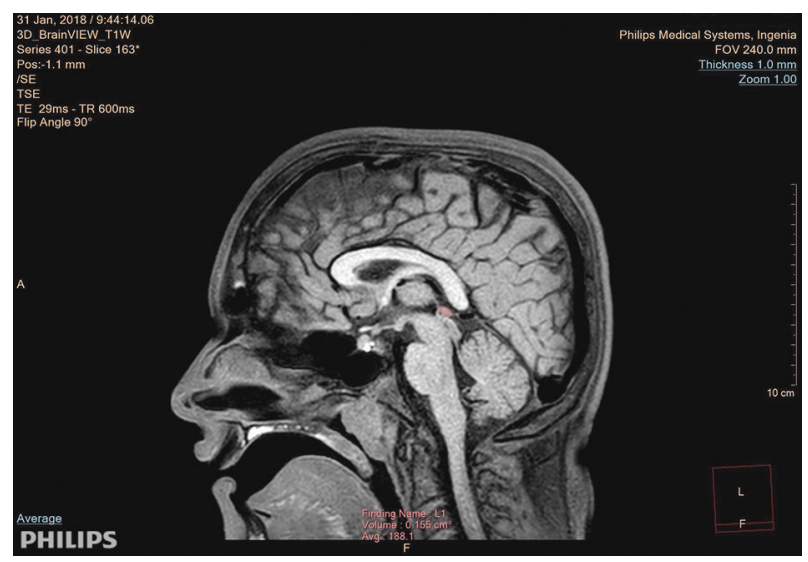

Figure 3. Pineal gland volume of a 16-year-old male patient measured using the tumour tracking method

under the 2 ROC curves. The $\chi^{2}$ test was used to analyse the number of patients with cysts and the patients' gender by group. Statistical significance level was taken as 0.05 , and SPSS 21 and MedCalc 16 demo versions were used in the analyses.

\section{Results}

Age and gender were homogenously distributed between the 2 study groups ( $p=0.688$ for age, $p=0.650$ for gender) (Table 1). Although the mean of the PPV was lower in the ASD patient group than in the control group, no significant difference was found between the 2 groups in terms of mean pineal cyst volume $(p<0.001, p=0.940$, respectively). The mean TPGV was lower in the patients with ASD than in the control group for both the MMV and TT techniques ( $p<0.001$ for both). The average PPV-MMV/

Table 1. Age and gender distribution of the autism spectrum disorder (ASD) patient and control groups

\begin{tabular}{|l|c|c|c|}
\hline Parameter & ASD & Control & $p$ \\
\hline Age (mean \pm SD) & $9.08 \pm 4.44$ & $8.82 \pm 4.54$ & $0.688^{\mathrm{a}}$ \\
\hline Gender, $n(\%)$ & \multicolumn{3}{|l}{} \\
\hline Female & $26(21.7)$ & $20(24.4)$ & $0.650^{\mathrm{b}}$ \\
\cline { 1 - 3 } Male & $94(78.3)$ & $62(75.6)$ & \\
\hline
\end{tabular}

astudent's $t$-test, ${ }^{b} \chi^{2}$ test

\begin{tabular}{|l|c|c|c|}
\hline Parameter & ASD $(n=120)$, mean \pm SD & Control $(n=82)$, mean \pm SD & $p$ \\
\hline PPV-MMV $\left(\mathrm{mm}^{3}\right)$ & $91.96 \pm 45.09$ & $139.22 \pm 42.41$ & $<0.001^{\mathrm{a}}$ \\
\hline PCV-MMV $\left(\mathrm{mm}^{3}\right)$ & $56.90 \pm 24.32$ & $57.38 \pm 27.38$ & $0.940^{\mathrm{a}}$ \\
\hline TPGV-MMV $\left(\mathrm{mm}^{3}\right)$ & $120.26 \pm 37.63$ & $155.26 \pm 35.93$ & $<0.001^{\mathrm{a}}$ \\
\hline TPGV-TT $\left(\mathrm{mm}^{3}\right)$ & $134.34 \pm 40.82$ & $167.29 \pm 37.29$ & $<0.001^{\mathrm{a}}$ \\
\hline Number of patients with a pineal cyst $(\mathrm{n})$ & $60(50.0 \%)$ & $21(25.6 \%)$ & $0.001^{\mathrm{b}}$ \\
\hline PPV-MMV/TPGV-MMV rate & $0.77 \pm 0.26$ & $0.90 \pm 0.19$ & $<0.001^{\mathrm{a}}$ \\
\hline
\end{tabular}

aStudent's $t$-test, ${ }^{b} \chi^{2}$ test, ASD - autism spectrum disorder, PPV - pineal parenchyma volume, PCV - pineal cyst volume, TPGV - total pineal gland volume, MMV - multimodality viewer, TT - tumour tracking 


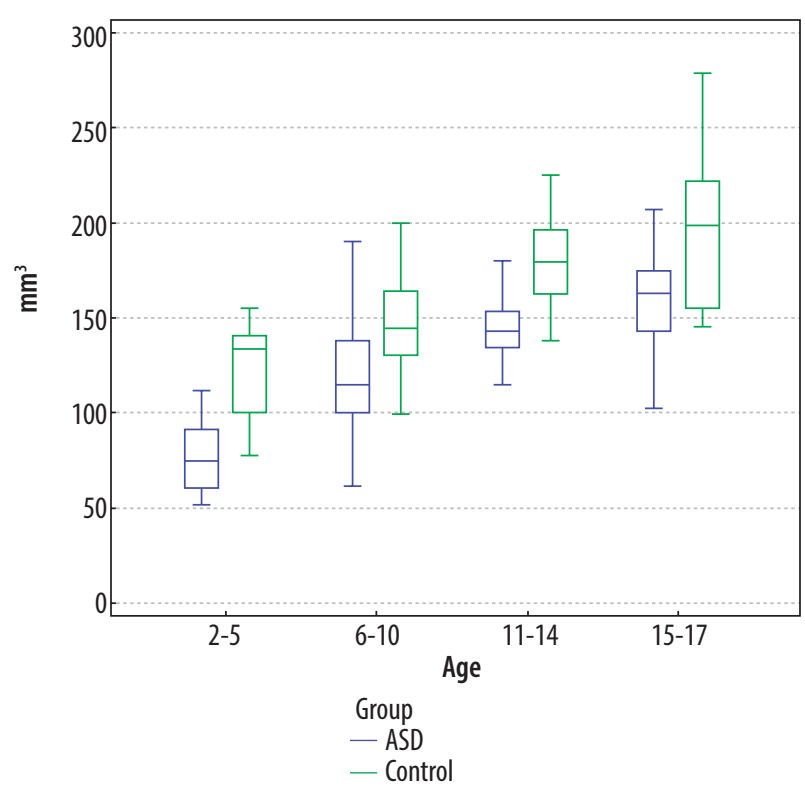

Figure 4. Comparison of the total pineal gland volume measurements of the autism spectrum disorder patients and the controls in all age groups using the multimodality viewer (MMV) technique ( $p=0.014$ for 15 -17-year-old age group; MMV, $p<0.001$ for all the other age groups)

Table 3. Analysis of the relationship between total pineal gland volume and age using both techniques

\begin{tabular}{|l|c|c|c|}
\multicolumn{2}{|c|}{} & TPGV-MMV $\left(\mathrm{mm}^{3}\right)$ & TPGV-TT $\left(\mathrm{mm}^{3}\right)$ \\
\hline \multirow{2}{*}{ ASD-Age } & $r$ & 0.824 & 0.791 \\
\cline { 2 - 4 } & $p$ & $<0.001$ & $<0.001$ \\
\hline \multirow{2}{*}{ Control-Age } & $r$ & 0.735 & 0.742 \\
\cline { 2 - 4 } & $p$ & $<0.001$ & $<0.001$ \\
\hline
\end{tabular}

$r$ - Pearson's correlation coefficient, ASD - autism spectrum disorder, TPGV - total pineal gland volume, MMV - multimodality viewer, TT - tumour tracking

TPGV-MMV ratio was lower in patients with ASD than in the control group $(p<0.001)$. The rate of patients with a pineal cyst was higher in the patients with ASD than in the control group $(p=0.001)$ (Table 2). The mean TPGV volume was statistically significantly lower for patients with ASD in all age groups in comparison to the control group, based on both measurement techniques (Figures 4 and 5).

A positive, strong linear relationship was found between age and TPGV for both the ASD patient group and the control group, for both techniques. As the patients' ages increased, the TPGV also increased (Table 3).

There was a very strong agreement between the TT and MMV techniques in terms of measuring the TPGV (intraclass correlation [95\% confidence interval] 0.961; range: 0.948-0.970).

According to the TPGV-MMV technique, the cut-off point for distinguishing between patients with ASD and the healthy control subjects was $\leq 125 \mathrm{~mm}^{3}$. Based on this value, the rate of detecting patients with ASD was $53.33 \%$, while the rate of detecting healthy children was $87.80 \%$. According to the MMV technique, the overall discrimination performance for

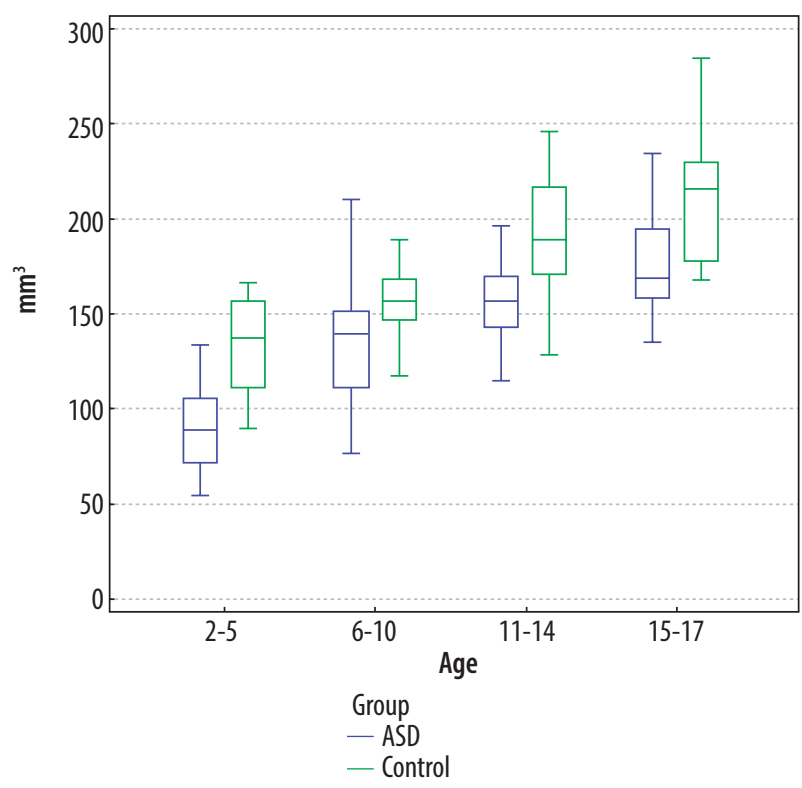

Figure 5. Comparison of the total pineal gland volume measurements of the autism spectrum disorder patients and the controls in all age groups using the tumour tracking (TT) technique ( $p=0.011$ for the 15 -17-year-old age group; TT, $p<0.001$ for all the other age groups)

the TPGV was $74.2 \%$. The cut-off point to differentiate patients with ASD from healthy patients was $\leq 150 \mathrm{~mm}^{3}$ when using the TPGV-TT technique. Based on this value, the rate of detecting patients with ASD was $62.5 \%$, while the rate of detecting healthy children was $70.73 \%$. The overall discrimination performance of the TPGV-TT technique was 71.3\%.

However, this value was not statistically significant. There was no difference in the TPGV measurements between the 2 techniques in terms distinguishing patients with ASD from healthy individuals $(p=0.056$ ) (Table 4, Figure 6).

\section{Discussion}

According to our measurements obtained using MMV and TT, the TPGV was lower in patients with ASD than in the control group $(\mathrm{p}<0.001)$. Moreover, the PPV and PPV-MMV/TPGV-MMV ratio were also found to be lower in the patients with ASD than in the control group $(p<0.001$ for both).

In the $16^{\text {th }}$ century, René Descartes defined the pineal gland as the "place of the soul" or the "organ of the psychophysiological control" [9]. The pineal gland is responsible for the synthesis of serotonin and N-N-dimethyltryptamine during the day and melatonin synthesis at night, with the circadian rhythm [10]. Although melatonin is known for its regulatory role in the circadian rhythm, it is also a powerful antioxidant, it has anti-inflammatory properties, and it helps regulate immune response and synaptic plasticity $[11,12]$.

The pineal gland is situated on the sagittal line between the posterior commissure and the dorsal habenular commissure. It is about 5-9 $\mathrm{mm}$ in length and $1-5 \mathrm{~mm}$ in width, and it has a solid, pinecone shaped and a red-grey colour $[9,13]$. In mammals, the size of the pineal gland depends on the cli- 
Table 4. ROC curve analysis of MMV and TT to predict the paediatric autism spectrum disorder (ASD)

\begin{tabular}{|l|c|c|c|c|c|} 
& AUC $(95 \% \mathrm{Cl})$ & Cut off, $\mathrm{mm}^{3}$ & SE $(95 \% \mathrm{CI})$ & SP $(95 \% \mathrm{Cl})$ & $p$ \\
\hline MMV & $0.742(0.676-0.801)$ & $\leq 125$ & $53.33(44.0-62.5)$ & $87.80(78.7-94.0)$ & $<0.001$ \\
\hline TT & $0.713(0.645-0.774)$ & $\leq 150$ & $62.50(53.2-71.2)$ & $70.73(59.6-80.3)$ & $<0.001$ \\
\hline Difference between areas & $0.029(-0.001$ to 0.061$)$ & & & & 0.056 \\
\hline
\end{tabular}

$p=\mathrm{ROC}$ (receiver operating characteristic curves) analysis, AUC - area under curve, $\mathrm{SE}$ - sensitivity, SP - specificity, $\mathrm{Cl}$ - confidence interval

mate, the geographic location, and the light stimulus activity [14]. The pineal gland consists of follicles and pinealocytes and glial tissue. The pinealocytes form the pineal parenchyma and the astrocytes dominate the glial tissue [15]. As the PPV increases, the amount of melatonin secreted increases in direct proportion. The PPV only contains pinealocytes. The more parenchymal calcifications, gliosis, and cysts, the more dysfunctional the secretory activities of the gland [16].

In ASD, the physiological levels of melatonin and/or melatonin derivatives are generally below average and are associated with autistic behaviour. In ASD, this might be due to the abnormalities in the genes associated with melatonin. Treatment with melatonin supplementation significantly improves sleep time and sleep delay in ASD [17,18]. Maruani et al. [19] found that both early-morning melatonin level and pineal gland volume were lower in patients with ASD in comparison to the controls. Another study found that the PPV was linearly correlated with the plasma concentrations of melatonin in humans [16]. That study showed similar differences between individuals with 24-hour melatonin per pineal tissue volume [16]. Examining the relationship between MRI volumetry and melatonin levels facilitates the characterization of the secretory capacity of the pineal gland. This is especially seen in diseases that include a disturbed circadian melatonin rhythm, such as schizophrenia, Parkinson's disease, sleep disorders, and aging [20-22]. We found the TPGV in patients with ASD to be low, in accordance with the literature, using both the MMV and TT methods. A brain imaging study in autistic patients reported abnormalities such as an increase in total brain volume and cerebellar abnormality [23].

Several rigorous radiological studies have been conducted on PGV in normal and abnormal cases. Autistic children can be calmed using mild anaesthesia when performing noninvasive imaging studies, such as brain MRIs [24]. A comprehensive study on pineal volume in healthy young adults reported that the mean pineal volume was $89.21 \pm$ $28.78 \mathrm{~mm}^{3}$ in male adults without cysts, and the true pineal volume in healthy adults was $94.2 \pm 40.65 \mathrm{~mm}[3,25]$. A statistically valuable study showed that the mean pineal volume was highest in the 31-40-year-old age group (158.34 \pm 43.17 $\mathrm{mm}^{3}$ ), the lowest mean volume was in the 41-50-year-old age group $\left(109.90 \pm 37.50 \mathrm{~mm}^{3}\right)$, in the under 30 -year-old age group the pineal volume was $131.47 \pm 41.42 \mathrm{~mm}^{3}$, and in the over 50-year-old age group the pineal volume was 124.97 $\pm 39.79 \mathrm{~mm}^{3}$ [26].

Pineal calcifications are considered to be hormonally inactive [27]. This is common in autopsies and computed tomography, where it is seen in more than $66 \%$ of all cases

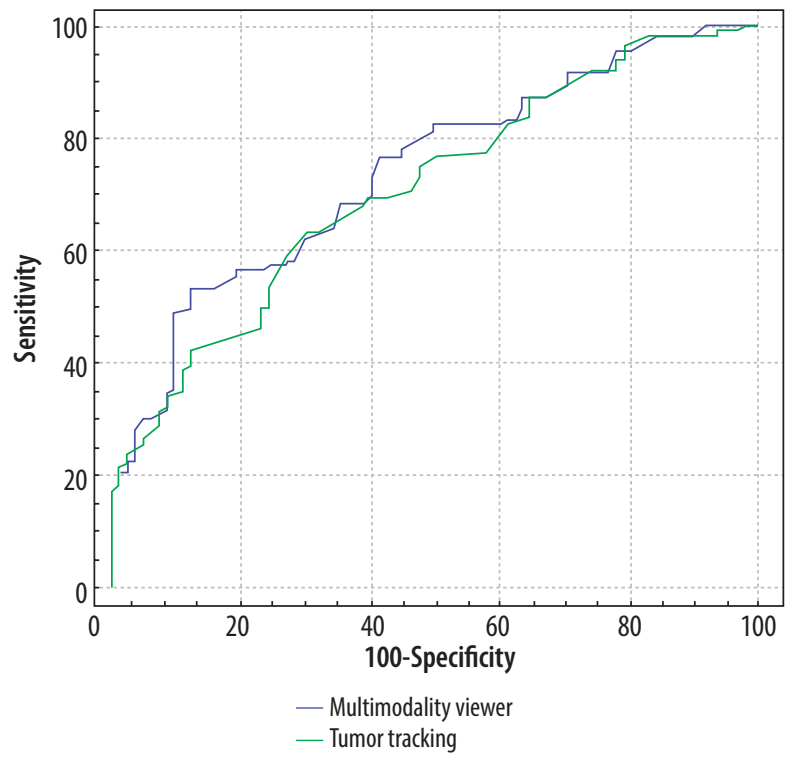

Figure 6. ROC curve analysis of multimodality viewer and tumour tracking for predicting paediatric patients with autism spectrum disorder

$[28,29]$. The effect of pineal calcifications on melatonin secretion is controversial. Kunz et al. [30] found no correlation between the degree of calcification and 24-hour sulphatoxymelatonin; however, they found a correlation between noncalcified pineal tissue and 24-hour sulphatoxymelatonin. Due to this controversial situation, we did not include patients with calcification in our study.

The prevalence of pineal cysts was found to be $0.6 \%$ in children and $2.6 \%$ in adults [31]. Pineal cysts can be detected by chance using 3-T MRI. Furthermore, PGV tends to increase with age [32]. In an MRI study in Iceland, the median volume of the pineal gland was found to be $207 \mathrm{~mm}^{3}$. This study also reported that the prevalence of cysts in this gland was $59 \%$ and the prevalence of calcifications was $20 \%$ [33]. Sumida et al. [7] reported that the size of the pineal gland increased from birth until 2 years of age and remained constant from the ages of 2 to 20 years. In our study, the rate of patients with a pineal cyst was significantly higher in patients with ASD (50\% vs. $25.6 \%$ ), while no difference in the cyst volumes was observed between the patients with ASD and the control group ( $p=0.001, p=0.940$, respectively). We also observed that the pineal volume increased with age in children with ASD.

This study has some limitations. First, the study population was small because we could not include patients with pineal calcification. Second, the study only included a volumetric analysis; moreover, we did not consider the relationship 
between PGV and melatonin. However, we also evaluated the parenchyma volume associated with direct melatonin release. Thirdly, the effect of antipsychotic drugs used by patients was not taken into consideration. Finally, we included patients with pineal cysts, but it was useful to compare the presence and volume of cysts in patients with ASD and the subjects in the control group.

\section{Conclusions}

Our study was the first work in the literature to show that autistic children had significantly lower total and parenchymal pineal gland volumes in comparison to normal children, using 2 different measurement methods (MMV and TT). We found the rate of pineal cysts was signifi- cantly higher in the children with ASD than in the healthy controls. We showed that the pineal volume in healthy children or children with ASD is dependent on age. This study also provides the normal distribution rate for the volumes of normal pineal glands in healthy children and autistic children aged between 2 and 17 years. We believe that further clarification and investigation of the abnormal findings we detected in ASD is warranted; in the future, more comprehensive and detailed studies will provide beneficial results for the pathogenesis of ASD patients.

\section{Conflict of interest}

The authors report no conflict of interest.

\section{References}

1. Yenkoyan K, Grigoryan A, Fereshetyan K, et al. Advances in understanding the pathophysiology of autism spectrum disorders. Behav Brain Res 2017; 331: 92-101.

2. Cohen S, Conduit R, Lockley SW, et al. The relationship between sleep and behavior in autism spectrum disorder (ASD): a review. J Neurodev Disord 2014; 6: 44.

3. Rossignol DA, Frye RE. Melatonin in autism spectrum disorders: a systematic review and meta-analysis. Dev Med Child Neurol 2011; 53: 783-792.

4. Geoffray MM, Nicolas A, Speranza M, et al. Are circadian rhythms new pathways to understand Autism Spectrum Disorder? J Physiol Paris 2016; 110: 434-438.

5. Emet M, Ozcan H, Ozel L, et al. A review of melatonin, its receptors and drugs. Eurasian J Med 2016; 48: 135-141.

6. Shen MD, Kim SH, McKinstry RC, et al. Increased extra-axial cerebrospinal fluid in high-risk infants who later develop autism. Biol Psychiatry 2017; 82: 186-193.

7. Sumida M, Barkovich AJ, Newton TH. Development of the pineal gland: measurement with MR. AJNR Am J Neuroradiol 1996; 17: 233-236.

8. Pu Y, Mahankali S, Hou J, et al. High prevalence of pineal cysts in healthy adults demonstrated by high-resolution, noncontrast brain MR imaging. AJNR Am J Neuroradiol 2007; 28: 1706-1709.

9. Nichols DE. N,N-dimethyltryptamine and the pineal gland: separating fact from myth. J Psychopharmacol 2018; 32: 30-36.

10. Pfeffer M, Korf HW, Wicht H. Synchronizing effects of melatonin on diurnal and circadian rhythms. Gen Comp Endocrinol 2018; 258: 215-221.

11. Malow BA, Findling RL, Schroder CM, et al. Sleep, growth, and puberty after two years of prolonged-release melatonin in children with autism spectrum disorder. J Am Acad Child Adolesc Psychiatry 2021; 60: 252-261.

12. El-Ansary A, Hassan WM, Daghestani M, et al. Preliminary evaluation of a novel nine-biomarker profile for the prediction of autism spectrum disorder. PLoS One 2020; 15: e0227626.

13. Patel S, Rahmani B, Gandhi J, et al. Revisiting the pineal gland: a review of calcification, masses, precocious puberty, and melatonin functions. Int J Neurosci 2020; 130: 464-475.
14. Tan DX, Xu B, Zhou X, et al. Pineal calcification, melatonin production, aging, associated health consequences and rejuvenation of the pineal gland. Molecules 2018; 23: E301.

15. Koshy S, Vettivel SK. Varying appearances of calcification in human pineal gland: a light microscopic study. J Anat Soc India 2001; 50: 17-18.

16. Nölte I, Lütkhoff AT, Stuck BA, et al. Pineal volume and circadian melatonin profile in healthy volunteers: an interdisciplinary approach. J Magn Reson Imaging 2009; 30: 499-505.

17. Jenabi E, Ataei S, Bashirian S. Evaluation of drug interventions for the treatment of sleep disorders in children with autism spectrum disorders: a systematic review. Korean J Pediatr 2019; 62: 405-409.

18. Cuomo BM, Vaz S, Lee EAL, et al. Effectiveness of sleep-based interventions for children with autism spectrum disorder: a meta-synthesis. Pharmacotherapy 2017; 37: 555-578.

19. Maruani A, Dumas G, Beggiato A, et al. Morning plasma melatonin differences in autism: beyond the impact of pineal gland volume. Front Psychiatry 2019; 10: 11.

20. Pandi-Perumal SR, Srinivasan V, Spence DW, et al. Role of the melatonin system in the control of sleep: therapeutic implications. CNS Drugs 2007; 21: 995-1018.

21. Mayo JC, Sainz RM, Tan DX, et al. Melatonin and Parkinson's disease. Endocrine 2005; 27: 169-178.

22. Pacchierotti C, Iapichino S, Bossini L, et al. Melatonin in psychiatric disorders: a review on the melatonin involvement in psychiatry. Front Neuroendocrinol 2001; 22: 18-32.

23. Boddaert N, Zilbovicius M. Functional neuroimaging and childhood autism. Pediatr Radiol 2002; 32: 1-7.

24. Ross AK, Hazlett HC, Garrett NT, et al. Moderate sedation for MRI in young children with autism. Pediatr Radiol 2005; 35: 867-71.

25. Sun B, Wang D, Tang Y, et al. The pineal volume: a three-dimensional volumetric study in healthy young adults using 3.0 T MR data. Int J Dev Neurosci 2009; 27: 655-660.

26. Golan J, Torres K, Staśkiewicz GJ, et al. Morphometric parameters of the human pineal gland in relation to age, body weight and height. Folia Morphol (Warsz) 2002; 61: 111-113. 
27. Schmid HA, Requintina PJ, Oxenkrug GF, et al. Calcium, calcification, and melatonin biosynthesis in the human pineal gland: a postmortem study into age-related factors. J Pineal Res 1994; 16: 178-183.

28. Hasegawa A, Ohtsubo K, Mori W. Pineal gland in old age; quantitative and qualitative morphological study of 168 human autopsy cases. Brain Res 1987; 409: 343-349.

29. Daghighi MH, Rezaei V, Zarrintan S, et al. Intracranial physiological calcifications in adults on computed tomography in Tabriz, Iran. Folia Morphol 2007; 66: 115-119.

30. Kunz D, Schmitz S, Mahlberg R, et al. A new concept for melatonin deficit: on pineal calcification and melatonin excretion. Neuropsychopharmacology 1999; 21: 765-772.
31. Sener RN. The pineal gland: a comparative MR imaging study in children and adults with respect to normal anatomical variations and pineal cysts. Pediatr Radiol 1995; 25: 245-248.

32. Whitehead MT, Oh CC, Choudhri AF. Incidental pineal cysts in children who undergo 3-T MRI. Pediatr Radiol 2013; 43: 1577-1583.

33. Sigurdardottir LG, Markt SC, Sigurdsson S, et al. Pineal gland volume assessed by MRI and its correlation with 6-Sulfatoxymelatonin levels among older men. J Biol Rhytms 2016; 31: 461-469. 